\title{
Longitudinal Trajectories of Health Related Quality of Life in Danish Family Members of Individuals with Severe Brain Injury
}

\author{
Anne Norup, ${ }^{1}$ Daniel J. Snipes, ${ }^{2}$ Lars Siert, ${ }^{1}$ Erik Lykke Mortensen, ${ }^{3}$ \\ Paul B. Perrin, ${ }^{2}$ and Juan Carlos Arango-Lasprilla ${ }^{4}$ \\ ${ }^{1}$ RUBRIC Research on Brain Injury rehabilitation Copenhagen, Department of \\ Neurorehabilitation, Traumatic Brain injury Unit, Copenhagen University Hospital, Glostrup, \\ Denmark \\ ${ }^{2}$ Department of Psychology, Virginia Commonwealth University, Richmond, VA, USA \\ ${ }^{3}$ Institute of Public Health and Center for Healthy Aging, University of Copenhagen, Copenhagen, \\ Denmark \\ ${ }^{4}$ University of Deusto, IKERBASOUE, Basque Foundation for Science, Bilbao, Spain
}

\begin{abstract}
Sant research has examined health-related quality of life (HRQoL) in family $\checkmark$ members of patients with severe brain injury, even less has been done in Scandinavian countries, and none has examined this construct longitudinally. The current study therefore used multilevel modelling to investigate the trajectories of HRQoL in 94 Danish family members of patients with severe brain injury at five time points, beginning at the patient's stay in a neuro intensive care unit through one year after injury. The family members' HRQoL scores significantly and strongly increased over time, and Role Limitations - Emotional scores were higher when patients had high Rancho Los Amigos Scale scores at admission to early intensive rehabilitation in hospital. These results suggest that the acute and sub-acute periods after brain injury are an extremely difficult time psychologically for many families, and family-based mental health interventions during the acute and sub-acute phases are critical, especially for families who have a patient with severe deficits.
\end{abstract}

Keywords: international, TBI caregivers, family systems, severe brain injury, longitudinal trajectories, health related quality of life

Traumatic brain injury (TBI) is a critical health issue internationally, affecting nearly 10 million individuals each year (Hyder, Wunderlich, Puvanachandra, Gururaj, \& Kobusingye, 2007). The World Health Organisation (WHO) estimates that deaths and disabilities due to traumatic brain injury will surpass those of many other diseases by the year 2020 (Hyder et al., 2007). The hallmark symptoms of TBI are

Address for correspondence: Anne Norup, PhD, RUBRIC Research Unit on BRain Injury Rehabilitation Copenhagen, Department of Neurorehabilitation, Glostrup, Denmark 
deficits in cognitive (Brooks, 1984), psychosocial (Cunningham \& Jones, 1999), behavioural/emotional, and physical functioning (Lynch, 1986). The relative invisibility of TBI (e.g., cognitive deficits rather than physical scarring) has led to the view of TBI as a 'silent epidemic' (Langlois, Rutland-Brown, \& Wald, 2006).

International research has illustrated that particular global regions have unique characteristics that may contribute to the prevalence, causes, and course of TBI. For example, Latin American and Caribbean regions have extremely high rates due to road traffic injuries, with Sub-Saharan Africa trailing closely behind (Hyder et al., 2007). Because of these statistics, research on TBI in these regions has begun to gain momentum (e.g. Arango-Lasprilla et al., 2008, 2011). However, other regions and countries are greatly affected by TBI as well. The annual cost of TBI in Europe is estimated at 33 billion Euros (Wittchen et al., 2011), and TBI incidence in Europe is increasing due to road traffic injuries, just as in low and middle-income countries (European Commission, 2011). In Denmark in particular, the focus of this article, road traffic injuries are the main cause of TBI (Engberg \& Teasdale, 2001; Engberg, Liebach, \& Nordenbo, 2006).

As a result of the deficits associated with severe brain injury, afflicted individuals often require the regular support of informal caregivers, most of whom tend to be family members (Knight et al., 1998). This sometimes involves providing literally a lifetime of care to the injured person (Sinnakaruppan \& Williams, 2001). Therefore, adjusting to and sustaining the caregiver role can be emotionally taxing for family members. An abundance of literature has documented the negative psychosocial outcomes associated with caregiving for a person with brain injury including high levels of stress, depression, anxiety, anger and isolation, as well as financial issues and poor social functioning (Knight et al., 1998, Arango-Lasprilla et al., 2008; Marsh, Kersel, \& Havill, 1998; Rotondi, Sinkule, Balzer, Harris, \& Moldovan, 2007).

When considering the burden of caregiving more holistically, the process of caregiving can wear away at family members' overall quality of life (Arango-Lasprilla et al., 2008). Quality of life is defined as the personal evaluation of one's physical, psychological, or social well-being (Chronister, Chan, Sasson-Gelman, \& Chiu, 2010). A similar construct identified in the literature and highly relevant to family members is health-related quality of life (HRQoL), which addresses aspects of daily functioning that are affected by one's own physical and mental health (Testa \& Simonson, 1996). HRQoL is often measured in studies that examine rehabilitation and health care, however, it is less studied among relatives of patients with brain injury, although extremely appropriate (McPherson, Pentland \& McNaughton, 2000; Chronister et al., 2010; Arango-Lasprilla et al., 2011). To further underscore the dearth of literature in this area, no research has longitudinally examined HRQoL among TBI family members. It is critical to study how HRQoL either improves or declines over time in this population, and what factors influence that trajectory so that interventions can be created to improve family members' HRQoL after brain injury.

Even scarcer than research examining HRQoL in family members, is research looking at families coping with a relative's brain injury in Denmark. Few studies have been published on this population, which is unfortunate given Denmark's comprehensive public health care system, where treatment and care do not depend on personal health insurance. Only two Danish studies have been published addressing HRQoL in family 
members of patients with severe brain injury, both of which reported on cross-sectional sub-samples from the current longitudinal sample. The more recent study explored this construct while patients were in a neuro intensive care unit (Norup, Welling, Qvist, Siert \& Mortensen, 2012), and the other upon patients' admission to early intensive rehabilitation in hospital (Norup, Siert \& Mortensen, 2010). Both studies identified low HRQoL in family members, as well as high anxiety and depression, and these variables were associated with patients' level of consciousness and functioning.

One other research study on families affected by brain injury in Denmark found that in comparison to families affected by diabetes, brain injury families reported more parental stress and family dysfunction, and spouses of the person with brain injury reported more depression and lower marital satisfaction than the diabetes group (Kieffer-Kristensen \& Teasdale, 2011). This finding suggests a very robust effect on family members of brain injury survivors in Denmark, even greater than that for diabetes.

Because of the paucity of research examining relatives' HRQoL, and the lack of research examining this construct longitudinally or in Denmark, the current study used multilevel modelling to investigate the trajectories of Danish family members' HRQoL beginning at the patient's stay in a neuro intensive care unit through one year after injury. Specifically, this study investigates whether relatives' HRQoL changes over time and whether these trajectories vary as a function of brain injury severity.

\section{Method}

\section{Participants}

Relatives were included when patients were admitted for intensive neurorehabilitation in hospital and consented to participate in the study. This consent was documented by a neuropsychologist at admission to the unit. The family decided which relative should participate if more relatives were present at the time of enrolment. About half of the total sample was included in the acute setting (T1). The sample included in the acute setting has been described thoroughly elsewhere (Norup et al., 2012).

Ninety-four family members of patients with severe brain injury in Denmark were recruited to participate in this study. Relatives were included if they were a child ( $\geq 18$ years old), parent, spouse/cohabitant, sibling or boy-/girlfriend (not cohabitant). Family-member participants were excluded who did not speak Danish or who had a psychiatric diagnosis or a progressive brain disease (e.g. dementia). Most were females $(74.5 \%)$, and the majority of relatives were cohabiting with the individuals with brain injury at the time of injury $(59.6 \%)$. Most relatives were parents $(42.6 \%)$ or spouses $(34.0 \%)$, and the remaining were children $(13.8 \%)$, siblings $(3.2 \%)$, aunts $(2.1 \%)$, or non-married romantic partners $(4.3 \%)$. Most relatives were working full time at the time of injury (81.9\%). Family members had a mean age of $48.80(\mathrm{SD}=12.80)$.

All patients had severe brain injury and had been admitted to intensive neurorehabilitation. All patients with TBI or non-traumatic brain injury (NTBI) must have had a GCS score between 3-9 (if adults or under age 5) or between 3-11 (if between age 5-15). Or, patients with a GCS above 9 were included who had severe focal neurological deficits such as aphasia, hemi paresis, or agitation. Patients had all completed neurosurgery (if applicable) and were respiratory stable. 


\section{Procedure}

Family members completed the primary outcome measure the SF-36 at four or five different assessment points depending on time of enrolment: while the person with brain injury was (a) in the neuro intensive care unit (T1), (b) at admission to early intensive rehabilitation (T2), (c) at discharge from rehabilitation (T3), (d) at 3 months post-discharge (T4), and (e) at one year post-injury (T5).

The majority of the patients were male $(70.2 \%)$, and most had sustained a TBI $(72.3 \%)$, while the others had sustained a NTBI. The NTBIs were caused by cardiac arrest $(4.3 \%)$, spontaneous intracranial hemorrhage $(4.3 \%)$, subarachnoid hemorrhage $(6.4 \%)$, infarction $(9.6 \%)$, tumour $(2.1 \%)$, or meningitis $(1.1 \%)$. The patients had a mean age of $38.31(S D=20.17)$.

This study was approved by the Committees on Biomedical Research Ethics of the Capital Region of Denmark (H-KF-311150) as well as by the Danish Data Protection Agency (2007-41-0583).

\section{Measures}

Short Form-36 (SF-36). The SF-36 (Stewart \& Ware, 1992) is a 36-item, widely used self-report measure of health-related quality of life (HRQoL). The SF-36 has been used both in individuals with TBI (Guilfoyle et al., 2010) and in TBI caregivers (Arango-Lasprilla et al., 2011; McPherson et al., 2000). For the purpose of this study, only four subscales were used that focus on relatives' psychosocial functioning: Role Limitations - Emotional (performance of role as affected by emotional factors), Social Functioning, Mental Health and Vitality. Scores on each subscale range from 0-100, with higher scores indicating better HRQoL. Family members' scores were evaluated in terms of gender according to the Danish norms (Bjorner et al., 1997).

Glasgow Coma Scale (GCS). The GCS measures the degree of consciousness in individuals with brain injury (Teasdale $\&$ Jennett, 1974), and the assessment is made by a trained physician. This assessment focuses on a number of factors including eye movement, verbal comprehension, and motor ability, which together provide a total score of responsiveness. Scores on this measure range from 3 (not conscious, indicative of severe brain injury) to 15 (full consciousness). In this study, GCS scores were assessed at admission to early intensive rehabilitation.

Rancho Los Amigos Scale (RLAS). The RLAS (Rancho Los Amigos National Rehabilitation Center, 2012) measures the degree of cognition (e.g., thinking and memory skills) as an individual awakes from a coma, and the assessment is made by a trained neuropsychologist. Scores on this measure range from 1 (unresponsive) to 8 (cognitive functioning that is automatic and appropriate). RLAS scores were assessed at admission to early intensive rehabilitation.

Functional Independence Measure (FIM). The FIM (Granger \& Hamilton, 1990) is a widely used method of assessing activities of daily living among individuals with a disability. The FIM consists of 18, 7-point items and has six subscales: Self Care, Sphincter Control, Transfer Capability, Locomotion, Communication, and Social Cognition. In the current study, only the total score was used. FIM scores were 


\section{TABLE 1}

Demographics

\begin{tabular}{|c|c|c|c|}
\hline & Patient $(\mathrm{n}=94)$ & & Family members $(n=94)$ \\
\hline & Mean (SD) & & Mean (SD) \\
\hline Age & $38.31(20.17)$ & & $48.80(12.80)$ \\
\hline & $n(\%)$ & & n (\%) \\
\hline Gender, males & $66(70.2 \%)$ & & $24(25.5 \%)$ \\
\hline Cohabiting at ti & & $56(59.6 \%)$ & \\
\hline Relationship Sp & & & $32(34.0 \%)$ \\
\hline & & & $40(42.6 \%)$ \\
\hline Ch & & & $13(13.8 \%)$ \\
\hline Sik & & & $3(3.2 \%)$ \\
\hline & & & $6(6.4 \%)$ \\
\hline
\end{tabular}

assessed by a trained physio- or occupational therapist at admission to early intensive rehabilitation.

\section{Data analyses}

Descriptive statistics were used to present demographics: results are presented as percentages and as means with standard deviations. One sample t-tests were used to compare the sample and the reference population.

Multilevel models were analysed to examine longitudinal changes in relatives' scores on the four psychosocial subscales of the SF-36 over the five data collection points. Additionally, these trajectories were examined to determine if they varied as a function of patients' GCS, RLAS, or FIM scores at admission to early intensive rehabilitation. A benefit of using MLM is the way in which missing data is handled. These MLM analyses used full information maximum likelihood (FIML) to estimate missing data in each model. This is a common rationale for performing MLM, as traditional repeated measures analyses of variance procedures eliminate participants with missing data at any time point. In these MLMs, time was treated as a continuous variable and was used to calculate a total slope for each dependent variable across time points. Because these analyses were conducted with a priori hypotheses, their results are presented without corrections for Type-I error. The statistical software used were SPSS version 19.0.

\section{Results}

Means on each scale at each time point was calculated, and the scores of the sample were significantly impaired compared to reference population (Bjorner et al., 1997) at all time points. The mean scores of the four scales are given in Table 2.

On all four scales administered the sample improved during the study period. 


\section{TABLE 2}

SF-36 Mean scores, standard deviations (SD) and range over time

\begin{tabular}{|c|c|c|c|c|c|c|c|c|c|c|c|c|}
\hline & \multicolumn{3}{|c|}{ RE } & \multicolumn{3}{|c|}{ SF } & \multicolumn{3}{|c|}{ VT } & \multicolumn{3}{|c|}{$\mathrm{MH}$} \\
\hline & $\mathrm{n}$ & Mean (SD) & Range & $\mathrm{n}$ & Mean (SD) & Range & $\mathrm{n}$ & Mean (SD) & Range & $\mathrm{n}$ & Mean (SD) & Range \\
\hline T1 & 38 & $27.19(30.86)^{* * *}$ & $0-100$ & 45 & $53.06(29.69)^{* * *}$ & $0-100$ & 44 & $28.64(18.94)^{* * *}$ & $0-75$ & 44 & $33.18(18.29)^{* * *}$ & $0-100$ \\
\hline T2 & 72 & $20.37(27.72)^{* * *}$ & $0-100$ & 86 & $53.77(28.89)^{* * *}$ & $0-100$ & 83 & $39.71(18.79)^{* * *}$ & $4-92$ & 84 & $32.50(19.07)^{* * *}$ & $0-75$ \\
\hline T3 & 68 & $34.31(35.95)^{* * *}$ & $0-100$ & 70 & $65.36(27.76)^{* * *}$ & $0-100$ & 67 & $41.34(18-98)^{* * *}$ & $0-95$ & 70 & $57.14(19.69)^{* * *}$ & $0-92$ \\
\hline T4 & 55 & $58.18(36.97)^{* * *}$ & $0-100$ & 59 & $70.34(26.96)^{* * *}$ & $12.5-100$ & 58 & $51.21(23.76)^{* * *}$ & $5-100$ & 57 & $66.10(18.73)^{* * *}$ & $16-100$ \\
\hline T5 & 58 & $62.64(40.50)^{* * *}$ & $0-100$ & 58 & $80.39(24.12)^{* *}$ & $25-100$ & 58 & $55.09(23.20)^{* * *}$ & $5-100$ & 58 & $70.14(18.81)^{* * *}$ & $28-100$ \\
\hline
\end{tabular}

Time points T1-T5 refers to: T1; in the neuro intensive care unit, T2: at admission to early intensive rehabilitation, T3: at discharge from rehabilitation, T4: 3 months post-discharge, T5: 1 year post-injury. $\mathrm{RE}=$ Role Emotional, $\mathrm{SF}=$ Social Function $\mathrm{VT}=$ Vitality, $\mathrm{MH}=$ Mental Health

$* \mathrm{p}<0.05, * * \mathrm{p}<0.01, * * * \mathrm{p}<0.001$ indicating level of significance compared to Danish reference population. 


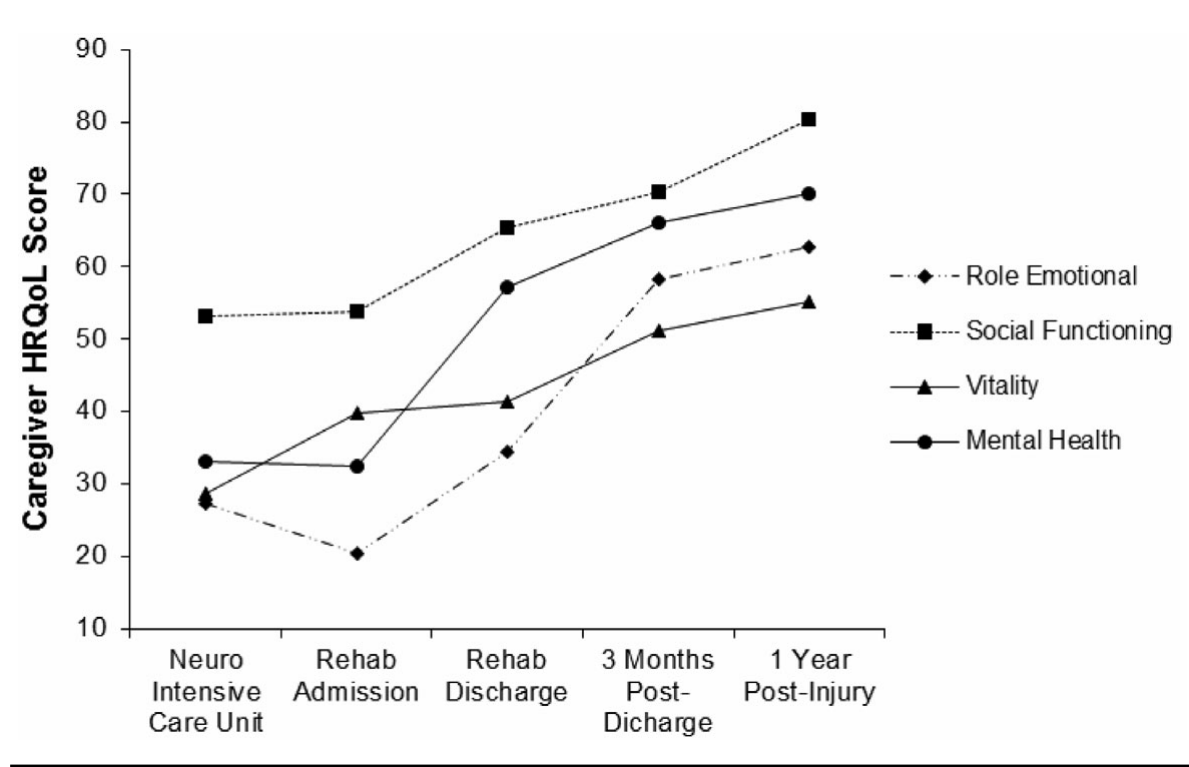

\section{FIGURE 1}

Caregiver health related quality of life scores over time.

\section{Linear Trajectories of Relatives' HRQoL over Time}

Four multilevel models were run for each of the four SF-36 psychosocial subscales. These models identified statistically significant upward linear trends for Role Limitations - Emotional $[b=11.81, t(291)=7.61, p<.001,95 \%$ CI: 8.75, 14.86], Social Functioning $[\mathrm{b}=7.43, t(318)=6.35, p<.001,95 \%$ CI: $5.14,9.72]$, Vitality $[\mathrm{b}=$ $6.22, t(310)=7.05, p<.001,95 \%$ CI: 4.48, 7.95], and Mental Health $[\mathrm{b}=11.30$, $t(311)=13.44, p<.001,95 \%$ CI: 9.64, 12.95]. These findings suggested that relatives' HRQoL scores for each of these variables increased significantly and strongly over time (Figure 1).

\section{Influences on Trajectories of Relatives' HRQoL trajectories}

A series of additional models were run for each of the four SF-36 psychosocial subscales in order to determine whether the level of these trajectories were influenced by patients' FIM, RLAS, or GCS scores at admission to early intensive rehabilitation. With RLAS, FIM, and GCS scores included in the model, RLAS at admission significantly predicted Role Limitations - Emotional trajectories $[b=7.93, t(53.58)=$ $2.35, p=.02,95 \%$ CI: 1.16, 14.71]. However, FIM $[b=-.07, t(49.60)=-.33, p=$ $.75]$ and GCS $[b=-1.72, t(52.64)=-1.05, p=.30]$ scores did not. RLAS $[b=2.88$, $t(47.19)=0.98, p=.33]$, FIM $[b=-.08, t(48.62)=-.42, p=.68]$, and GCS scores $[b=-1.66, t(50.56)=-1.17, p=.25]$ were not predictive of Social Functioning trajectories. GCS $[b=-.03, t(57.88)=-.03, p=.98]$, RLAS $[b=.40, t(53.58)=$ $.18, p=.86]$, and FIM $[b=-.05, t(54.15)=-.32, p=.75]$ were also not significant predictors of Vitality trajectories. Finally, GCS $[b=-.44, t(53.35)=-.48, p=.64]$, 
FIM $[b=-.06, t(50.19)=-.48, p=.63]$, and RLAS $[b=1.13, t(48.62)=.59, p=$ .56] were all not significant predictors of Mental Health trajectories.

\section{Discussion}

The purpose of this study was to investigate whether family members' HRQoL changed during the first year after brain injury and whether these trajectories varied as a function of injury severity. The results showed that HRQoL improved significantly in family members during the first year after injury. Moreover, family members of patients with higher RLAS scores at admission to early intensive rehabilitation showed significantly higher in Role Limitations - Emotional scores.

No other longitudinal studies have investigated HRQoL in family members of individuals with severe brain injury. However, cross-sectional data have revealed the draining impact of brain injury on relatives' HRQoL in the early as well as chronic phases of brain injury (Norup et al., 2010; Norup et al., 2012; McPherson et al., 2000; Arango-Lasprilla, 2011). McPherson et al. (2000) used the Short Form-36 (SF-36) to estimate HRQoL in a sample of 70 family caregivers 15-18 months after injury. Caregivers reported a non-significant trend toward lower HRQoL compared to a reference population on the following subscales: Role LimitationsPhysical, Social Functioning, Role Limitations-Emotional and Mental Health. Arango-Lasprilla and colleagues (2011) also compared SF-36 scores between a healthy control group and TBI caregivers who had been providing care for at least three months, although the time after injury varied. Caregivers had significantly lower scores on the Role-Emotional, Vitality, Social Functioning, Mental Health, Bodily Pain and General Health subscales compared to the control group who were matched on age, gender, marital status and education.

As mentioned in the Introduction, Norup and colleagues have published two cross-sectional studies (Norup et al., 2010; Norup et al., 2012) reporting significantly impaired HRQoL in a cross-sectional sub-sample of the present sample. The studies were conducted in the early phases of rehabilitation: in the neuro intensive care unit and at admission to early intensive rehabilitation. The Danish samples reported lower means than what was reported by McPherson et al. (2000) and Arango-Lasprilla et al. (2011), indicating lower HRQoL in the Danish samples. An important difference, however, is that the Danish results of the cross-sectional investigations as well as the current longitudinal study all took place within the first year after injury, whereas McPherson's and Arango-Lasprilla's studies employed a longer time interval since injury. Whether the reported differences are due to the different time intervals since injury or differences between the study populations is difficult to disentangle. Moreover, comparing the results of these studies is complicated by large differences between health care systems across the continents. In Denmark, the public health care system provides all patients with an opportunity for rehabilitation in hospitals based on individual needs regardless of their health insurance. Consequently, all patients receive early intensive rehabilitation in hospitals, and families do not struggle with insurance companies, public authorities, etc. in order to obtain care and rehabilitation for their family member with brain injury. In other countries such as the United States or many Latin American countries, the amount and duration of rehabilitation after brain injury 
is a reflection of the family's personal health insurance, and rehabilitation is to a lesser extent grounded in the specific rehabilitation needs of the patient. Because all Danish patients with severe TBI are provided with rehabilitation during the first months after injury, no families are forced to provide early neurorehabilitation care at home or to place their loved one in a nursing home due to a lack of other opportunities. Therefore, Danish families are not faced with the same responsibilities soon after a brain injury as are families in other countries involving physical care and assistance with activities of daily living. This might suggest that strain on family members in Denmark is lower or less complex than in other countries, which consequently could indicate better HRQoL. However, as no other studies have investigated HRQoL in the early phases of rehabilitation, this conclusion remains speculative.

Furthermore, the emotional distress caused by the consequences of brain injury such as family role change, increased isolation and personality changes in the individual with brain injury is assumed to be equal across countries, as high frequencies of anxiety and depression have been reported for Danish populations (Norup et al., 2010, 2011, 2012). Thus, despite the differences between health care systems in different countries, Danish families faced with brain injury do experience emotional distress and impaired HRQoL. Even though this study reported a significant improvement in HRQoL during the first year after injury, the mean scores of the sample one year after injury were still significantly lower compared to the Danish norms (Bjorner et al., 1997). Despite the comprehensive public health care system in Denmark, relatives of patients with severe brain injury do report significantly impaired HRQoL during the first year after injury.

The study also found that for brain injury patients with higher RLAS scores at admission (higher levels of cognition), family members had higher Role-Emotional subscale scores over time. Previous cross-sectional studies have indicated similar associations with higher levels of functioning in patients being related to better mental health in family members (Norup et al., 2010; Pielmaier, Walder, Rebetez \& Maercker, 2011). However, previous results have been limited by the innate problems of cross-sectional studies, as these are not able to provide any information regarding changes over time or the direction of causality (Flanders, Lin, Pirkle \& Caudill, 1992). As the current study showed, family members of brain injury patients with low levels of cognitive functioning at hospital admission are likely to experience lower HRQoL over time, and their family members may therefore be heavily in need of mental health services.

\section{Clinical Implications}

It is vital that rehabilitation professionals be aware of the emotionally draining and long-lasting effects of brain injury on family members. These effects may be especially pronounced in family members of patients with a low level of consciousness in the early phases of rehabilitation. As such, families of patients with low consciousness might need more psychological support during early rehabilitation and over time because doing so may improve the trajectory of HRQoL especially in this at-risk group of families. Low HRQoL experienced by family members may have important long-term consequences for the family with respect to employment and overall quality of life. Early detection and necessary support may prevent continued impairment in HRQoL, 
although such an assertion needs to be supported by future research on interventions for family members of brain injury survivors.

It is important for health care professionals and organisations to develop and test structured support systems based on clinical experience and empirical data that can adequately meet the needs of families. This is important in both the early phases of rehabilitation as well as later in rehabilitation because as this study shows, the effects of brain injury on family members continue for a long time post-injury. Support systems for families can include professionals working in the same rehabilitation setting or links to other organisations specialising in this support, whether public or voluntary. Effective strategies linking multiple services in the different phases of rehabilitation may decrease family members' strain and distress and improve the quality of informal care that they are able to provide after the patient's hospital discharge. As a result, a comprehensive family-focused approach in brain injury rehabilitation may be more effective for both the patient and family.

\section{Study Limitations}

Despite the current study's contributions to the scientific literature, it has several limitations worth noting. The single-centre design of this study and the fact that patients received comprehensive rehabilitation both warrant caution when generalising the results to other centres and other countries, especially those without government health care. The intensive rehabilitation services that patients received in this study might have improved family members' HRQoL, as rehabilitation services in other centres or countries may not be as comprehensive. However, this study found that the Danish HRQoL means were actually lower than the ones reported in the previous Mexican and New Zealand samples, although the time of assessment after injury was different. Unfortunately, the current study did not investigate whether the increases in family member's HRQoL continued to a level comparable to the reference population or whether the reported increase stabilises after several years. This would likely be a fruitful area for future research.

Secondly, the heterogeneity of the sample may limit generalisability as well. The family members of both NTBI and TBI patients were included, and one could argue that family members of patients with different etiologies face dissimilar situations that influence their HRQoL. However, this limitation can be tempered by the fact that in the current study, injury etiology was not associated with any HRQoL measure at any of the five time points. A third issue that might limit generalisability is the manner in which family members were selected to participate: when more than one relative was present at the time of enrolment, family members themselves decided who would participate. In many cases, one of several eligible relatives volunteered, and this choice may have resulted in bias, perhaps including the most resilient or extroverted relative. A fourth possible limitation is that it was not possible to collect family member baseline HRQoL scores before brain injury. Family members may have had low HRQoL before their loved one acquired a brain injury, but this would be up to speculation.

Fifthly, no control group was included in the study, and consequently how HRQoL evolves over time in healthy controls is unknown. However, previous research has 
supported the stability of SF-36 in a healthy population (Obidoa, Reisine \& Cherniack, 2010) indicating that the increase reported in our study is not due to test-retest effects.

Sixthly, we only applied four of the eight subscales of the SF-36. Because family members were enroled at a very vulnerable and distressed time, we chose to limit the burden on participants of having to fill out additional scales. The same patterns found in this study may or may not emerge with the physical health subscales of the SF-36 or in other measures of mental health. A final limitation is that although data were collected at five time points, not all participants provided data at every time point. Attrition is often reported as a problem in TBI research (Corrigan et al., 2003), so this limitation in the current study is not unique to the current sample. This presents an obvious limitation of the study, but we made efforts to limit the effects of attrition on our findings by using multilevel models, which - in contrast to repeated measures ANOVA - makes it possible to include participants who did not participate in all five assessments.

\section{Future Research}

The lack of research describing the early effects of brain injury on family members as well as investigations on effects of early intervention is obvious. Future studies should focus on the early phases of rehabilitation, as this is a critical timeframe for the essential triangle in rehabilitation: patients, family members and professionals. Rehabilitation is a partnership between these three groups of individuals, and the HRQoL of family members is an aspect that cannot be overlooked in future research. Studies should also determine whether the increase in HRQoL found in this study continues after the first year post-injury and whether it ever reaches a level comparable to reference populations. This article has commented on the comprehensive public health care system in Denmark, and despite this comprehensiveness, family members of patients with severe brain injury still experience major impairments in HRQoL. Future studies should examine whether family members in health care systems such as Denmark's, as well as in other nations' health care systems, feel that their and the patient's needs are being met, and if not, studies on this topic will be critical in the creation of family interventions to meet those needs.

\section{References}

Arango-Lasprilla, J.C., Nicholls, E., Villaseñor Cabrera, T., Drew, A., Jiminez-Maldonado, M., \& Martinez-Cortes, M.L. (2011). Health-related quality of life in caregivers of individuals with traumatic brain injury from Guadalajara, Mexico. Journal of Rehabilitation Medicine, 43, 983-986.

Arango-Lasprilla, J.C., Ketchum, J.M., Dezfulian, T., Kreutzer, J.S., O’Neil-Pirozzi, T.M., Hammond, F., \& Jha, A. (2008). Predictors of marital stability 2 years following traumatic brain injury. Brain Injury, 22, 564-574.

Bjorner, J.B., Damsgaard, M.T., Watt, T., \& Bech, P. (1997). [Danish manual for SF-36. A healthrelated questionnaire]. Copenhagen: Medif.

Brooks, D.N. (1984). Cognitive deficits after head injury. In: Brooks, D.N., editor. Closed Head Injury: Psychological, social, and family consequences. Oxford: Oxford University Press, 148-178.

Chronister, J., Chan, F., Sasson-Gelman, E.J., \& Chiu, C.Y. (2010). The association of stresscoping variables to quality of life among caregivers of individuals with traumatic brain injury. NeuroRehabilitation, 27, 49-62. 
Corrigan, J.D., Harrison-Felix, C., Bogner, J., Dijkers, M., Terrill, M.S., \& Whiteneck, G. (2003). Systematic bias in traumatic brain injury outcome studies because of loss to follow-up.Archives of Physical Medicine and Rehabilitation, 84; 153-160.

Cunningham, J.M., Chan, F., \& Jones, J. (1999). Brain injury rehabilitation: a primer for case managers. In: Chan, F. \& Leahy, M.J. (Eds.). Health Care and Disability Case Management. Illinois: Vocational Consultants Press, 475-526.

European Commission: The International Initiative for Traumatic Brain injury Research (InTBIR). Retrieved October 8, 2012 from http://ec.europa.eu/research/health/medical-research/brainresearch/international-initiative_en.html

Engberg, A., \& Teasdale, T. (1998). Traumatic brain injury in children in Denmark: A national 15-year study. European Journal of Epidemiology, 14(2), 165-173.

Engberg, A., \& Teasdale, T. (2001). Traumatic brain injury in Denmark 1979-1996. A national study of incidence and mortality.European Journal of Epidemiology, 17(5), 437-442.

Engberg, A.W., Liebach, A., \& Nordenbo, A. (2006). Centralized rehabilitation after severe traumatic brain injury - a population-based study. Acta Neurologica Scandinavica, 133, 178-184.

Flanders, W.D., Lin, L., Pirkle, J.L., \& Caudill, S.P. (1992). Assessing the direction of causality in crosssectional studies. American Journal of Epidemiology, 135, 926-935.

Granger, C.V., \& Hamilton, B.B. (1990). UDS Report: The uniform data system for medical rehabilitation report on first admissions for 1990. American Journal of Physical Medicine Rehabilitation, $71,108-113$.

Guilfoyle, M.R., Seeley, H.M., Corteen, E., Harkin, C., Richards, H., Menon, D.K., \& Hutchinson, P.J. (2010). Assessing quality of life after traumatic brain injury: Examination of the short form 36 health survey. Journal of Neurotrauma, 27, 2173-2181.

Hyder, A., Wunderlich, C.A., Puvanachandra, P., Gururaj, G., \& Kobusingye, O.C. (2007). The impact of traumatic brain injuries: A global perspective. NeuroRehabilitation, 22(5), 341-353.

Kieffer-Kristensen, R., \& Teasdale, T.W. (2011). Parental stress and marital relationships among patients with brain injury and their spouses. Neurorehabilitation, 28(4), 321-330.

Knight, R.G., Devereux, R., \& Godfrey, H.P.D. (1998). Caring for a family member with a traumatic brain injury. Brain injury, 12, 467-481.

Langlois, J.A., Rutland-Brown, W., \& Wald, M.M. (2006). The epidemiology and impact of traumatic brain injury: A brief overview, The Journal of Head Trauma Rehabilitation, 21(5), 375-378.

Lynch, R.T. (1986). Traumatic brain injury: Implications for rehabilitation counseling. In: Riggar, T.F., Maki, D.R., Wolf, A.W. (Eds.). Applied Rehabilitation Counseling. New York: Springer, 262-270.

Marsh, N.V., Kersel, D.A., \& Havill, J.H. (1998). Caregiver burden at 1 year following severe Traumatic brain injury. Brain injury, 12, 1045-1059.

McPherson, K.M., Pentland, B., \& McNaughton, H.K. (2000). Brain injury - the perceived health of carers. Disability and Rehabilitation, 22, 683-689.

Norup, A., Siert, L., \& Mortensen, E.L. (2010). Emotional distress and quality of life in relatives of patients with severe brain injury: The first month after injury. Brain Injury, 24, 81-88.

Norup, A., Kristensen, K.S., Siert, L., Poulsen, I., \& Mortensen, E.L (2011). Neuropsychological support to relatives of patients with severe traumatic brain injury in the sub-acute phase. Neuropsychological Rehabilitation, 21, 306-321.

Norup, A., Welling, K.L., Qvist, J., Siert, L., \& Mortensen, E.L. (2012). Depression, anxiety and quality-of-life among relatives of patients with severe brain injury: The acute phase. Brain Injury, 5, 1192-2000.

Obidoa, C.A., Reisine, S.L., \& Cherniack, M. (2010). How does the SF-36 perform in healthy populations? A structured review of longitudinal studies. Journal of Social, Behavioral, and Health Sciences, 4, 30-48. 
Pielmaier, L., Walder, B., Rebetez, M.M., \& Maercker, A. (2011). Post-traumatic stress symptoms in relatives in the first weeks after severe traumatic brain injury. Brain Injury, 25, 259-265.

Rancho Los Amigos National Rehabilitation Center. (2012). The Rancho levels of cognitive functioning. Retrieved October 12, 2012, from http://www.rancho.org/Research_RanchoLevels.aspx

Rotondi, A.J., Sinkule, J., Balzer, K., Harris, J., \& Moldovan, R. (2007). A qualitative needs assessment of persons who have experienced traumatic brain injury and their primary family caregivers. Journal of Head Trauma Rehabilitation, 22(1), 14-25.

Sinnakaruppan, I.M., \& Williams, D.M. (2001). Family carers and the adult head-injured: a critical review of carers' needs. Brain Injury, 15, 653-672.

Stewart, A.L., \& Ware, J.E. (1992). Measuring Functioning and Well-Being: The Medical Outcomes Study Approach. Durham, NC: Duke University Press.

Teasdale, G.M., \& Jennett, B. (1974). Assessment of coma and impaired consciousness: A practical scale. Lancet, 2, 81-84.

Testa, M.A., \& Simonson, D.C. (1996). Assessment of quality-of-life outcomes. New England Journal of Medicine, 334, 835-840.

Wittchen, H.U., Jacobi, F., Gustavsson, J.R., Svensson, M., Jönsson, B., \& Steinhausen, H.C. (2011). The size and burden of mental disorders and other disorders of the brain in Europe 2010. European Neuropsychopharmacology, 21, 655-679. 